\title{
Study on the Characters of Phase-Shifted Fiber Bragg Grating in Asymmetric Perturbation and Its Application in Fiber Laser Acoustic Sensor
}

\author{
Yanjie ZHAO ${ }^{1}$, Jiasheng $\mathrm{NI}^{2 *}$, Faxiang $\mathrm{ZHANG}^{2}$, Zhiqiang $\mathrm{SONG}^{2}$, Luyan $\mathrm{LI}^{1}$, \\ Ting CHEN ${ }^{1}$, and Shuang $\mathrm{LI}^{1}$ \\ ${ }^{1}$ School of Science, Shandong Jianzhu University, Jinan 250101, China \\ ${ }^{2}$ Laser Institute, Qilu University of Technology (Shandong Academy of Sciences), Jinan 250014, China \\ *Corresponding author: Jiasheng NI E-mail: njsh51@163.com
}

\begin{abstract}
A $\pi$ phase-shifted fiber Bragg grating theoretical model is established, and the effects of an asymmetric and symmetrical perturbation field on a phase-shifted fiber Bragg grating are investigated in this paper. The trends of wavelength shifting caused by effective refraction index of phase shift grating in symmetric and asymmetric acoustic field are investigated in detail. Then, the fiber laser acoustic sensors packaged in asymmetric and symmetrical structures are designed and tested, respectively. The results show that the acoustic response of the wavelength of the distributed feedback (DFB) fiber laser (FL) in an asymmetric packaging structure is much more sensitive than in that in the symmetrical structure. The sensor packaged in the asymmetrical structure has a better low frequency $(0 \mathrm{~Hz}-500 \mathrm{~Hz})$ performance and a higher sensitivity than that in the symmetrical structure, and the sensitivity is improved about $15 \mathrm{~dB}$ in average and $32.7 \mathrm{~dB}$ in maximum. It provides a new method to improve the sensitivity of the fiber acoustic sensor.
\end{abstract}

Keywords: Fiber acoustic sensor; phase-shifted fiber Bragg grating; asymmetric perturbation

Citation: Yanjie ZHAO, Jiasheng NI, Faxiang ZHANG, Zhiqiang SONG, Luyan LI, Ting CHEN, and Shuang LI, "Study on the Characters of Phase-Shifted Fiber Bragg Grating in Asymmetric Perturbation and Its Application in Fiber Laser Acoustic Sensor," Photonic Sensors, 2018, 8(4): 351-357.

\section{Introduction}

Distributed feedback fiber lasers (DFB FLs) are used in the fiber laser acoustic sensor [1-4] and light source seeds [5-7] because of their high strain sensitivity, robust single frequency performance, low frequency noise, and multiplexing ability. It has attracted significant interest.

Some important properties of the DFB fiber laser have been researched recently, such as wavelength [6], linewidth [8], noise [5], and polarization [8,9]. When it is used as a light seed to produce a high power and narrow linewidth laser, the wavelength stability is the most important aim. The wavelength character of the DFB fiber laser determines the quality of the light system. On the contrary, a fiber laser acoustic sensor requires the wavelength to be highly sensitive to external perturbations. The bare fiber laser has relatively low acoustic pressure sensitivity, so the enhancement of the sensitivity is the main topic by coating the bare fiber with some materials or structures designs [10-14]. Kersey et al. [15] and Koo et al. [16] reported that a narrow line-width fiber laser was selected as the sensing

Received: 13 April 2018 / Revised: 23 July 2018

(C) The Author(s) 2018. This article is published with open access at Springerlink.com

DOI: $10.1007 / \mathrm{s} 13320-018-0510-0$

Article type: Regular 
element, which was exposed to the acoustic environment in modulating its emission wavelength proportionally to its amplitude. Foster et al. [17] reported that poor resistance to bending capacity meant that the DFB fiber laser only required a small lateral force to cause a large degree of bending. Then in 2011, they reported a bender bar-based packaging scheme and pasted a DFB fiber laser on a flexible micro-machined beam, whose length was much less than that of the laser on silicon wafer, and demonstrated an excellent sensitivity in the order of $2 \mathrm{~nm} / \mathrm{MPa}$ and the flat frequency response more than $5 \mathrm{kHz}$ [18]. In 2013, Unnikrishnan et al. [19] reported that they utilized a thin large diaphragm-type package with novel engineering for pre-stress arrangement, and obtained a sensitivity of $-160 \mathrm{~dB}$ ref $\mathrm{rad} / \mu \mathrm{Pa}$ and a flat response up to $5 \mathrm{kHz}$ of operation. Launay et al. [20] demonstrated a wideband mechanical amplification design with high acoustic sensitivity. However, the complex nature of these packaging schemes made their fabrication and assembling difficult and expensive.

Most of coatings on the DFB fiber laser are implemented by using some material wrapped tightly. But those coatings generally may damage the laser due to the differential strain. Hence, its usefulness as an effective packaging for acoustic sensors is not much tested [21]. Another method is to enhance the strain of the DFB fiber laser by increasing the force bearing area. But the size of the sensor will increase and its high frequency response will lose.

All of those researches are under the premise that the DFB fiber laser device is in a symmetric perturbation field. The performance of a DFB fiber laser in an asymmetric perturbation field has not yet been reported to the extent of our knowledge.

In this paper, a $\pi$ phase-shifted fiber Bragg grating theoretical model is established, and the effects of an asymmetric and symmetrical perturbation field on a distributed feedback fiber laser sensor are investigated. The trends of wavelength shifting caused by an effective refraction index of phase shift grating in symmetric and asymmetric acoustic field are investigated in detail. Then, DFB fiber acoustic sensors packaged in asymmetric and symmetrical structures are designed and tested, respectively.

\section{Theory and methods}

The configuration of a DFB fiber laser is shown in Fig. 1. It consists of a phase-shifted grating written on a short section of $\mathrm{Er}^{3+}$ doped fiber. Normally, the optical phase shift is $\pi / 2$ and located at the center of the grating. When the DFB fiber laser is optically pumped with a light of shorter wavelength, it will emit stimulated emission with a narrow bandwidth at the wavelength of the band pass peak. Due to having short effective cavity length, most DFB fiber lasers can readily achieve single mode operation and stable output. The specific wavelength and line width are determined by the transfer function of the gratings and get narrowed due to the emission bandwidth of the active fiber.

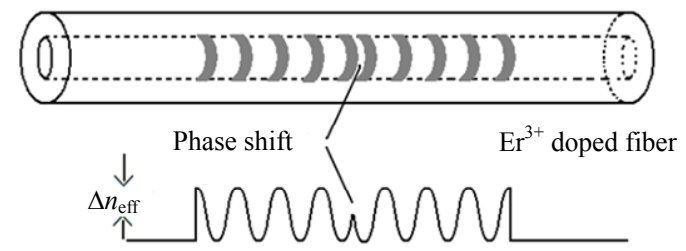

Fig. 1 DFB fiber laser configuration.

The transfer matrix method (TMM) is a commonly used tool for analyzing the reflection spectrum of fiber Bragg grating (FBG) and fiber phase-shifted grating $[22,23]$. TMM can be used to represent the solutions of the coupled-mode theory, e.g. to construct the solutions of multi-sectional (separated into $M$ sections in this paper) DFB structures within a phase-shifted regime between and facet reflectivity regimes at both ends [24]. By dividing the fiber grating into a large number of uniform pieces, the mode coupling of each piece can be expressed in a matrix form as 


$$
\mathbf{F}_{i}=\left[\begin{array}{cc}
\cosh (\gamma l)+i \frac{\hat{\sigma}}{\gamma} \sinh (\gamma l) & i \frac{k}{\gamma} \sinh (\gamma l) \\
-i \frac{k}{\gamma} \sinh (\gamma l) & \cosh (\gamma l)-i \frac{\hat{\sigma}}{\gamma} \sinh (\gamma l) \\
\gamma &
\end{array}\right]
$$

where $i$ is the number of each section, $0 \leqslant i \leqslant M$. $\gamma=\sqrt{k^{2}-\hat{\sigma}^{2}}, \hat{\sigma}$ is the direct current (DC) coupling efficiency, and $k$ is the alternating current (AC) coupling efficient, both of which are functions of effective refraction index $n_{\text {eff }}$ and grating pitch $\Lambda$. For a phase-shifted grating, a phase shift matrix is inserted at the location of the phase shift as

$$
\mathbf{F}_{\text {sint }}=\left[\begin{array}{cc}
\exp \left(-i \varphi_{i} / 2\right) & 0 \\
0 & \exp \left(i \varphi_{i} / 2\right)
\end{array}\right]
$$

where $\varphi_{i}$ is the phase shift value.

To simulate the spectrum of the DFB fiber laser, we set the number of sections as $n=150$, phase shift as $\varphi_{i}=\pi / 2$, and its position in the center as $n / 2=75$. The final transfer matrix can be determined as follows:

$$
\mathbf{F}_{\text {stift }}=\prod_{j=0}^{j=n / 2-1} \mathbf{F}_{j} \cdot \mathbf{F}_{\text {sidit }} \cdot \prod_{j=n / 2+1}^{j=n} \mathbf{F}_{j} \cdot
$$

The final result can be got by imposing the boundary conditions [24]. As Fig. 2 shows, the lasing window will be located at the center of the grating. The effective refraction index increment is expressed as $\delta_{n}$ caused by external disturbing. The wavelength of the DFB fiber laser $\lambda_{F L}$ is equal to the Bragg wavelength of the grating $\lambda_{\text {Bragg }}$ and also equal to the wavelength of the lasing window.

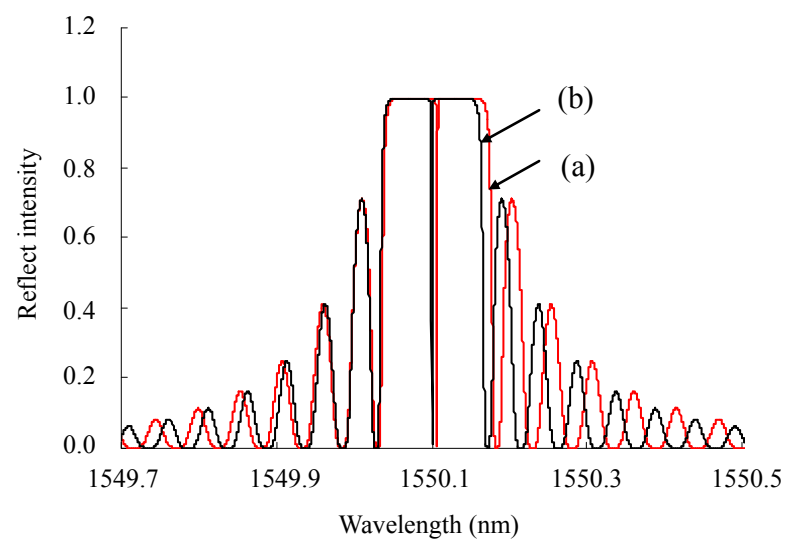

Fig. 2 Phase shift grating spectrum of the DFB fiber laser in a symmetric perturbation field $n_{\text {eff }}=1.449$ : (a) $\delta_{n}=0$ and (b) $\delta_{n}=0.2$.

\section{Results and discussion}

\subsection{Simulation results}

External perturbation will cause the DFB fiber laser to undergo dimensional and refractive index changing, which leads to a shift of lasing wavelength $\lambda_{F L}$ [25]. In the presence of acoustic fields, due to the elastic-optic effect and acoustic-optical effect, the effective refraction index of the grating will be changed by external perturbation, thereby the reflection spectrum of the grating will be changed together. In a homogeneous field (as Fig. 3 shows), two sides of the DFB fiber laser (separated by a $\pi / 2$ phase shift) are affected synchronously, which will make the effective refraction change synchronously. But in an asymmetric field (as Fig. 4 shows), two sides of the DFB fiber laser are affected asymmetrically. The results are completely different in these two conditions.

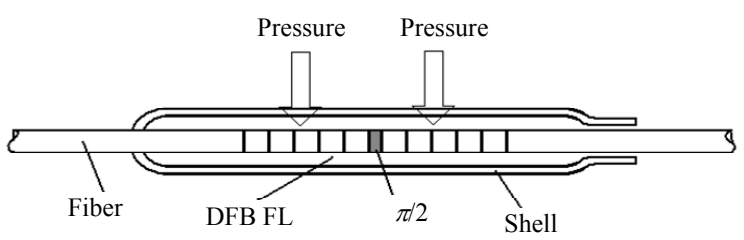

Fig. 3 Symmetrical structure of DFB fiber laser acoustic sensor.

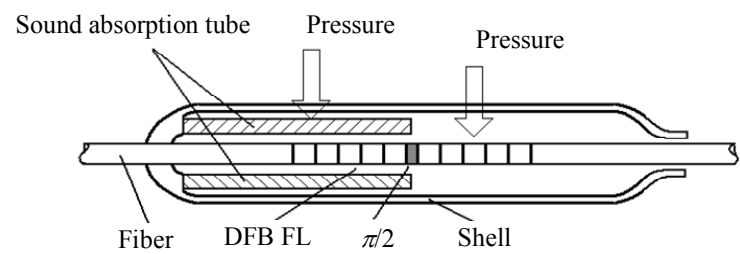

Fig. 4 Asymmetrical structure of DFB fiber laser acoustic sensor.

From Fig. 5 we can see that by increasing the effective refraction index increment $\delta_{n}$ from 0 to 0.2 , the wavelength of the fiber laser $\lambda_{F L}$ in a symmetrical field is changed from $1550.107 \mathrm{~nm}$ to $1550.094 \mathrm{~nm}$, which decreases about $13 \mathrm{pm}$. A linear relation between $\lambda_{F L}$ and $\delta_{n}$ is found, and the slope of the curve is -0.065 . In the asymmetrical field, by increasing $\delta_{n}$ from 0 to $0.0005, \lambda_{F L}$ is changed from $1550.107 \mathrm{~nm}$ to $1550.0375 \mathrm{~nm}$, which 
decreases nearly $70 \mathrm{pm}$. And a nonlinear relation between $\lambda_{F L}$ and $\delta_{n}$ is found (as Fig. 6 shows).

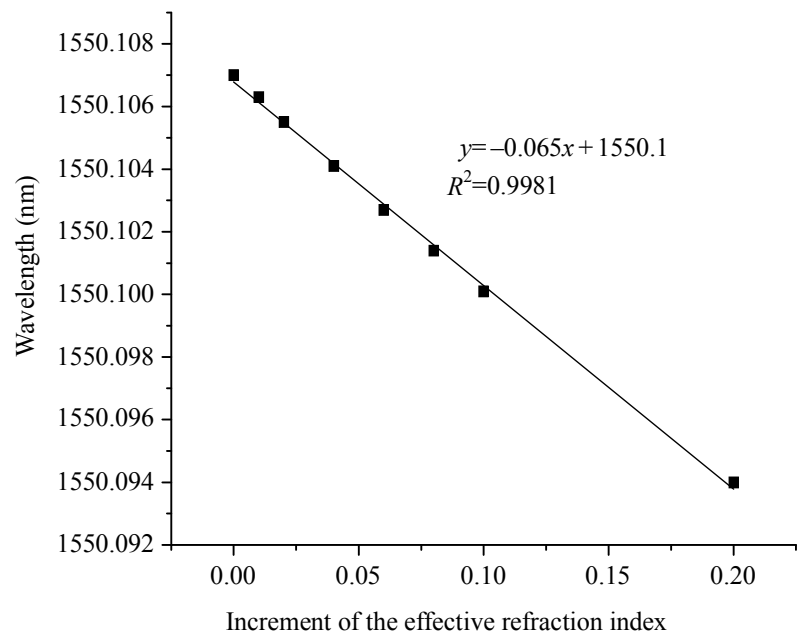

Fig. 5 Wavelength response curve in a symmetrical perturbation.

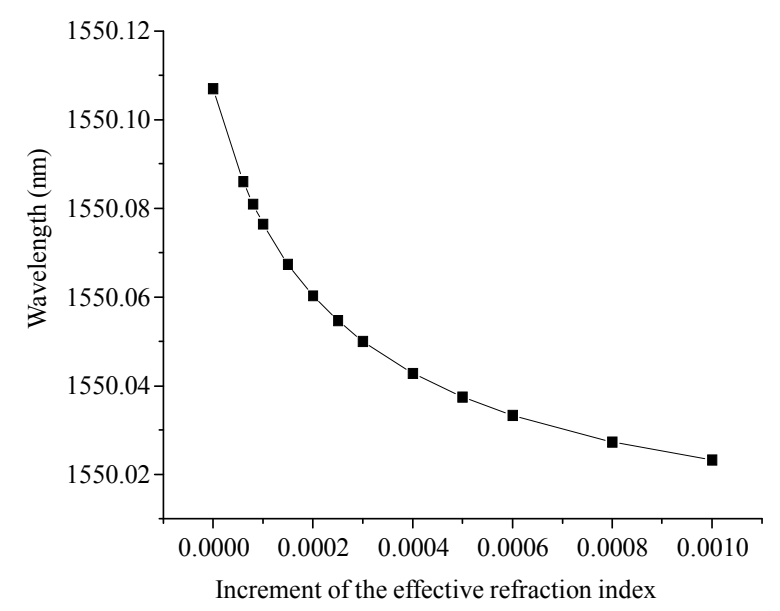

Fig. 6 Wavelength response curve in an asymmetrical perturbation.

It is well known that the wavelength of DFB fiber laser is sensitive to the axial strain $(\varepsilon)$, and the slope of $\lambda_{F L} / \varepsilon$ is positive, which is opposite to $\lambda_{F L} / \delta_{n}$. And axial strain is regarded as the main factors because it is obviously more effect. In general, $\delta_{n}$ can be neglected for its relatively smaller wavelength response than the axial strain in a uniform perturbation. But it can't be ignored in a non-uniform field such as asymmetrical perturbation discussed in this paper, because wavelength changes largely when the refractive index changes. Especially, when it is disturbed by an asymmetric horizontal force, the DFB fiber laser axial strain will become the secondary factor, while effective refraction index will be the leading factor in this case.

\subsection{Application in fiber laser acoustic sensor}

A polarization-dependent grating is generated by a vertically polarized ultraviolet (UV) scanning laser [26]. In the fabrication process, the phase shift is introduced by a movement of the phase mask to the fiber during beam scanning [27]. In this paper, we employ a UV laser (244 nm frequency-doubled harmonic Argon ion continuous wave laser) to write phase-shifted grating in the photosensitive $\mathrm{Er}^{3+}$ doped fiber (Nufern, the peak absorption at $1530 \mathrm{~nm}$ is $8 \mathrm{~dB} / \mathrm{m}$ ) by scanning UV beam across the phase mask (as Fig. 7 shows). The scanning speed of the UV laser is electrically controlled to obtain the desiring UV-induced index modulation amplitude. The phase mask is put on a piezoelectric transducer (PZT) moving stage with the nm-grade resolution and accuracy. The phase shift is introduced by an instantaneous movement of the phase mask to the fiber at the center of the phase-shifted grating, and the movement is $\Lambda / 4$ for a $\pi / 2$ phase shift.

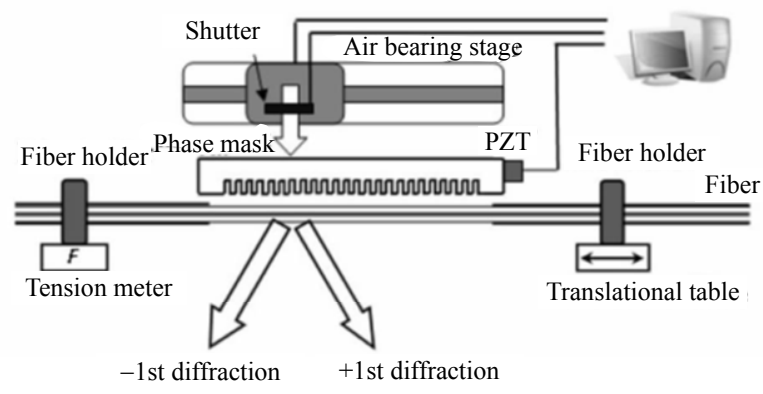

(a)

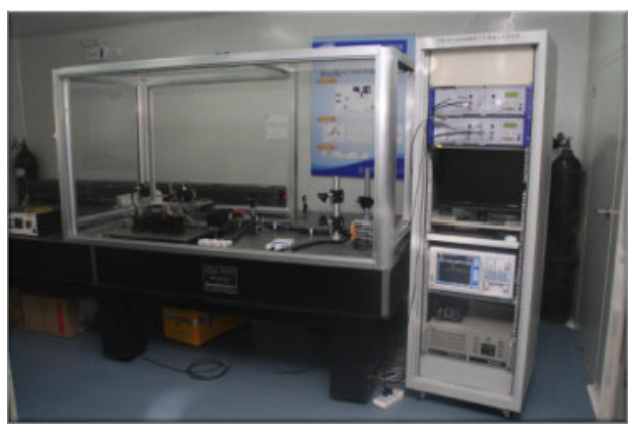

(b)

Fig. 7 Fabrication system of phase-shift grating using the phase mask: (a) structure diagram and (b) picture. 
As Fig. 8 shows, we design a test system for the DFB fiber laser acoustic sensors. The DFB fiber laser sensor is pumped by a $980 \mathrm{~nm}$ semiconductor laser. The interferometric interrogation method is adopted to realize a high resolution wavelength shift demodulation. The wavelength shift of the fiber laser is converted into a phase shift by using an unbalanced interferometer. The interferometer is well packaged because the isolation of the interferometer is very important to prevent environmental perturbations such as temperature, noise and vibration. Phase-generated carrier (PGC) and 3-by-3 coupler is employed in the system [28]. Optical phase demodulator (OPD4000, supplied by Optiphase Inc.) can demodulate sensor's response. And a reference probe is put near the fiber laser acoustic sensor to revise deviation of the acoustic source.

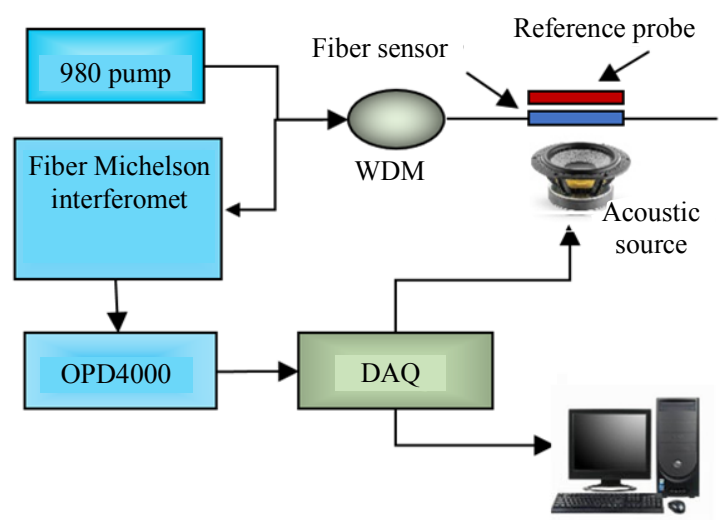

(a)

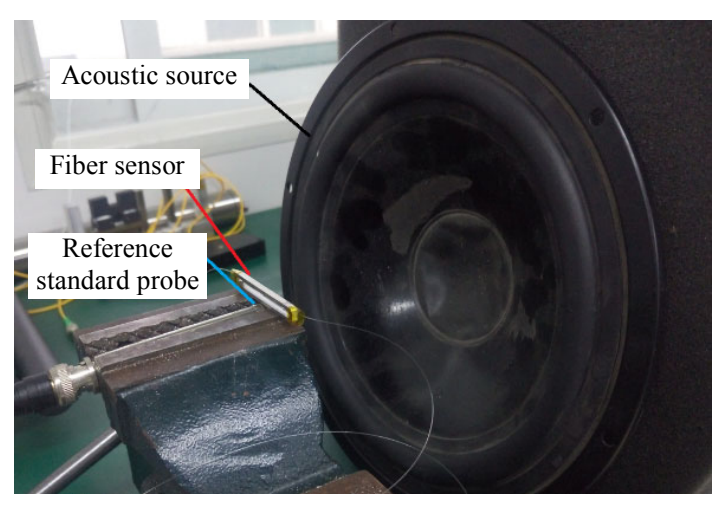

(b)

Fig. 8 Test system of DFB fiber laser acoustic sensor: (a) structure diagram and (b) picture.

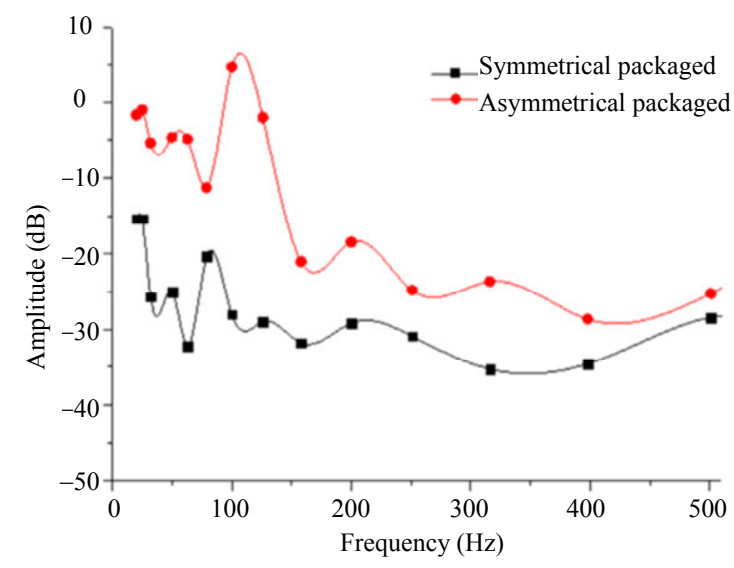

Fig. 9 Acoustic response of the DFB fiber laser acoustic sensor.

The DFB fiber laser acoustic sensors are packaged in the structures of Fig. 3 and Fig. 4, respectively. The test results are presented in Fig. 9. The test results show that the acoustic response of the acoustic sensor packaged in the asymmetrical structure has a better low frequency $(0 \mathrm{~Hz}-500 \mathrm{~Hz})$ performance and a higher sensitivity than that in the symmetrical structure. The sensitivity is improved about $15 \mathrm{~dB}$ in average and $32.7 \mathrm{~dB}$ in maximum. The resonance peak of the asymmetrical structure sensor is about $110 \mathrm{~Hz}$, while symmetrical structure sensor is about $80 \mathrm{~Hz}$.

\section{Conclusions}

We establish a $\pi$ phase-shifted fiber Bragg grating theoretical model and investigate the effects of an asymmetric and symmetrical perturbation field on a phase-shifted fiber Bragg grating in this paper, and found that the asymmetric acoustic field would bring a greater effect to wavelength shifting. It can be utilized to improve the sensitivity of the fiber laser acoustic sensor. To verify it, we design two fiber laser acoustic sensor package structures, which are in asymmetric and symmetrical, respectively. The test results show that the acoustic response of the acoustic sensor packaged in the asymmetrical structure has a better low frequency $(0 \mathrm{~Hz}-00 \mathrm{~Hz})$ performance and a higher sensitivity than that in the symmetrical structure. The sensitivity of the acoustic 
sensor is improved about $15 \mathrm{~dB}$ on average and $32.7 \mathrm{~dB}$ in maximum in the asymmetric packaging structure.

\section{Acknowledgment}

This work was supported by the Natural Science Foundation of Shandong Province of China (Grant No. ZR2016FB03), the National Natural Science Foundation of China (Grant No. 61705121\& No.21603122), and the Ph.D. Foundation of Shandong Jianzhu University (Grant No. XNBS1535).

Open Access This article is distributed under the terms of the Creative Commons Attribution 4.0 International License (http://creativecommons.org/licenses/by/4.0/), which permits unrestricted use, distribution, and reproduction in any medium, provided you give appropriate credit to the original author(s) and the source, provide a link to the Creative Commons license, and indicate if changes were made.

\section{References}

[1] L. N. Ma, Y. M. Hu, H. Luo, and Z. L. Hu, "DFB fiber laser hydrophone with flat frequency response and enhanced acoustic pressure sensitivity," IEEE Photonics Technology Letters, 2009, 21(17): 1280-1282.

[2] C. K. Kirkendall and A. Dandridge, "Overview of high performance fiber-optic sensing," Journal of Physics D Applied Physics, 2004, 37(18): 197-216.

[3] S. Foster, G. Cranch, J. Harrison, A. Tikhomirov, and G. Miller, "Distributed feedback fiber laser strain sensor technology," Journal of Lightwave Technology, 2017, 35(16): 3514-3530.

[4] W. Liu, L. N. Ma, Z. L. HU, Y. Feng, and H. Y. Yang, "Study of Rayleigh-backscattering induced coherence collapse in an asymmetric DFB FL sensor," Photonic Sensors, 2016, 6(3): 209-213.

[5] Y. J. Zhao, Q. P. Wang, J. Chang, J. S. Ni, C. Wang, Z. H. Sun, et al., "Suppression of the intensity noise in DFB fiber lasers by self-injection locking," Laser Physics Letters, 2012, 9(10): 739-743.

[6] F. F. Hou and M. Yang, "A novel dual-wavelength DFB fiber laser," Optik, 2013, 124(18): 3674-3677.

[7] S. Loranger, A. Tehranchi, H. Winful, and R. Kashyap, "Realization and optimization of phase-shifted distributed feedback fiber Bragg grating Raman lasers," Optica, 2018, 5(3): 295-302.
[8] J. S. Ni, Y. J. Zhao, C. Wang, G. D. Peng, T. Y. Liu, J. Chang, et al., "Research on linewidth characteristics and broadening mechanism of distributed feedback fiber laser," Acta Physica Sinica, 2012, 61(8): 084205-1-084205-6.

[9] Y. J. Zhao, Q. P. Wang, J. Chang, J. S. Ni, C. Wang, P. P. Wang, et al., "Linewidth narrowing and polarization control of erbium-doped fiber laser by self-injection locking," Laser Physics, 2011, 21(12): 2108-2111.

[10] J. A. Bucaro and H. D. Dardy, "Fiber optic hydrophone," The Journal of the Acoustical Society of America, 1997, 62(5): 1302-1304.

[11] D. J. Hill and G. A. Cranch, "Gain in hydrostatic pressure sensitivity of coated fiber Bragg grating," Electronics Letters, 1999, 35(15): 1268-1269.

[12] W. T. Zhang, Y. L. Liu, F. Li, and H. Xao, "Fiber laser hydrophone based on double diaphragms: theory and experiment," Journal of Lightwave Technology, 2008, 26(10): 1349-1352.

[13] J. Z. Zhang, X. L. Li, Q. Chai, Q. Q. Hao, Q. Li, W. M. Sun, et al., "Hydrophone based on intensity modulated DFB fiber laser," in Proceeding of IEEE Sensors, Kona, HI, USA, 2010, pp. 315-317.

[14] A. I. Azmi, I. Leung, X. B. Chen, S. L. Zhou, Q. Zhu, $\mathrm{K}$. Gao, et al., "Fiber laser based hydrophone system," Photonic Sensors, 2011, 1(3): 210-221.

[15] A. D. Kersey, K. P. Koo, and M. A. Davis, "Fiber optic Bragg grating laser sensors," SPIE, 1994, 2292: 102-112.

[16] K. P. Koo and A. D. Kersey, "Bragg grating based laser sensors systems with interferometric interrogation and wavelength division multiplexing," Journal of Lightwave Technology, 1995, 13(7): 1243-1249.

[17] S. Foster, A. Tikhomirov, M. Milnes, J. V. Velzen, and G. Hardy, "A fiber laser hydrophone," SPIE, 2005, 5855: 627-630.

[18] S. Foster, A. Tikhomirov, and J. V. Velzen, "Towards a high performance fiber laser hydrophone," Journal of Lightwave Technology, 2011, 29(9): 1335-1342.

[19] K. C. Unnikrishnan and P. Venugopalan, "Pressure compensated fiber laser hydrophone: modeling and experimentation," Journal of the Acoustical Society of America, 2013, 134(4): 2710-2718.

[20] F. X. Launay, R. Lardat, R. Bouffaron, G. Roux, and M. Doisy, "Static pressure and temperature compensated wideband fiber laser hydrophone," SPIE, 2013, 8794: 87940k-1-87940k-5.

[21] K. Vivek, R. Rajesh, C. V. Sreehari, S. Shamkumar, K. Shajahan, T. V. Praveen, et al., "A new approach of large diameter polymer-coated fiber laser hydrophone," Journal of Lightwave Technology, 
2017, 35(19): 4097-4104.

[22] P. G. Agrawal and S. Radic, "Phase-shifted fiber Bragg gratings and their application for wavelength demultiplexing," Photonics Technology Letters IEEE, 1994, 6(8): 995-997.

[23] A. Tehranchi, S. Loranger, and R. Kashyap, "Engineered pi-phase-shifted fiber Bragg gratings for efficient distributed feedback Raman fiber lasers," IEEE Journal of Quantum Electronics, 2018, 54(3): 1-7.

[24] T. Makino and J. Glinski, "Transfer matrix analysis of the amplified spontaneous emission of DFB semiconductor laser amplifiers," IEEE Journal of Quantum Electronics, 1988, 24(8): 1507-1518.

[25] S. W. Løvseth and K. Bløtekjær, "Contributions to wavelength shifts of DFB fiber lasers used as acoustic sensors in air," SPIE, 1998, 3483: 69-73.

[26] M. Ibsen, E. Ronnekleiv, G. J. Cowle, M. O. Berendt, O. Hadeler, M. N. Zervas, et al., "Robust high power $(>20 \mathrm{~mW})$ all-fibre DFB lasers with unidirectional and truly single polarisation outputs," in Proceeding of Conference of Lasers and Electro-Optics, Baltimore, MD, USA, 1999, pp. 245-246.

[27] L. Poladian, B. Ashton, W. E. Padden, A. Michie, and C. Marra, "Characterisation of phase-shifts in gratings fabricated by over-dithering and simple displacement," Optical Fiber Technology, 2003, 9(4): 173-188.

[28] G. A. Cranch, G. M. H. Flockhart, and C. K. Kirkendall, "Distributed feedback fiber laser strain sensors," IEEE Sensors Journal, 2008, 8(7): 1161-1172. 